\title{
Espécies de Adelpha Hübner, [1819] (Lepidoptera, Nymphalidae, Limenitidinae) ocorrentes no Rio Grande do Sul, Brasil
}

\author{
Rocco Alfredo Di Mare ${ }^{1}$ \\ José Augusto Teston² \\ Elio Corseuil ${ }^{3}$
}

\begin{abstract}
Species of Adelpha Hübner, [1819] (Lepidoptera, Nymphalidae, Limenitidinae) occurring in Rio Grande do Sul State, Brazil. Based on literature, collections and sampled butterflies, a list of twelve species of Adelpha Hübner occurring in Rio Grande do Sul State is presented, including host plants. Adelpha epizygis Fruhstorfer, [1916], Adelpha falcipennis Fruhstorfer, [1916], Adelpha goyama Schaus, 1902 and Adelpha isis (Drury, 1782) are new reports to Rio Grande do Sul. The species are illustrated and keyed.
\end{abstract}

KEYwords. Adelpha; host plants; inventory; Nymphalidae; taxonomy.

\section{INTRODUÇÃO}

O gênero Adelpha Hübner, [1819] formado por pouco menos de uma centena de espécies, com distribuição desde os Estados Unidos da América até o Uruguai e Argentina, é um dos mais complexos da família Nymphalidae.

Embora estas borboletas sejam bem conhecidas, existem poucos estudos de abrangência mais ampla (GodMAN \& SALVIN 1879-1901; FruHSTORFER 1907; Moss 1933; HALl 1938; HAYWARD 1950; Miller \& Miller 1970; Young 1974; Aiello 1984; Willmott \& Hall 1999). Além destes, há trabalhos que as citam para determinada região e/ou país (JÖRGENSEN 1921; DE Lesse 1967; Silva et al. 1968; BiezanKo et al. 1978; Devries 1985).

O primeiro estudo de revisão do grupo foi efetuado para as espécies da América Central (Godman \& S ALvin 1879-1901), sendo FrUHSTORFER (1907) o primeiro a revisar o gênero em sua totalidade. Muitas das espécies são semelhantes, de identificação difícil, existindo mais de duas centenas de nomes na literatura, incluídos erros de identificação, repetidos e combinados em inúmeros trabalhos, por várias décadas.
Em relação ao Rio Grande do Sul, os estudos com citações de Adelpha, são os de Weymer (1894), Mabilde (1896), BiezANKo \& Freitas (1938) e BieZanko (1949), que listam as espécies para o Estado ou para determinada região; já os de RONNA (1933), BieZANKo et al. (1949), BerTELs \& BAUCKE (1966) e SiLVA et al. (1968) relacionam as espécies com suas respectivas plantas hospedeiras e os de BiezANKo \& BAUCKe (1948) e BiEZANKO \& LinK (1972), os nomes populares de lepidópteros.

Este trabalho foi desenvolvido com o objetivo de contribuir para o conhecimento da entomofauna do Rio Grande do Sul, atualizar a nomenclatura e fornecer informações sobre a distribuição e habitat de espécies.

\section{MATERIALE MÉTODOS}

Foram realizadas coletas e examinadas as coleções: "Bertels", do Centro de Pesquisa Agropecuária de Clima Temperado, EMBRAPA, Pelotas (CAMB); Museu Entomológico Ceslau Biezanko, Faculdade de Agronomia Eliseu Maciel, Universidade Federal de Pelotas, Pelotas (MECB); Museu de Ciências Naturais, Universidade Católica de Pelotas,

\footnotetext{
1. Universidade Federal de Santa Maria, Centro de Ciências Naturais e Exatas, Departamento de Biologia, Campus Universitário, km 9 Faixa de Camobi, 97119-900 Santa Maria - RS, Brasil. Endereço eletrônico: ram13@terra.com.br

2. Pontifícia Universidade Católica do Rio Grande do Sul, Programa de Pós-Graduação em Biociências. Caixa Postal 1429, 90619-900 Porto Alegre - RS, Brasil. Endereço eletrônico: jateston@pucrs.br

3. Pontifícia Universidade Católica do Rio Grande do Sul, Faculdade de Biociências. Caixa Postal 1429, 90619-900 Porto Alegre - RS, Brasil. Endereço eletrônico: corseuil@pucrs.br
} 
Pelotas (MUCP); Museu Anchieta de Ciências Naturais, Porto Alegre (MAPA); "Mabilde", "Palombini” e "Ronna", Museu de Ciências Naturais, Fundação Zoobotânica do Rio Grande do Sul, Porto Alegre (MCNZ); Museu de Ciências e Tecnologia, Pontifícia Universidade Católica do Rio Grande do Sul, Porto Alegre (MCTP); Museu Ramiro Gomes Costa, Fundação Estadual de Pesquisa Agropecuária, Porto Alegre (MRGC); Setor de Entomologia, Faculdade de Agronomia da Universidade Federal do Rio Grande do Sul, Porto Alegre (FASE); "D'Almeida", Departamento de Zoologia, Universidade Federal do Paraná, Curitiba (DZUP); Coleção Rocco Alfredo Di Mare, Santa Maria (CRAM).

A identificação das espécies foi realizada com base em Mabilde (1896), Fruhstorfer (1907), Hayward (1950), De VRies (1985) E Brown JR. (1992). As espécies e subespécies estão listadas em ordem alfabética, com a indicação da coleção onde o exemplar está depositado e os autores que as registraram para o Estado; a ocorrência está indicada pelos municípios onde foram capturadas e, quando for o caso, a observação em reservas e/ou parques ecológicos, entre parênteses; também são indicadas plantas hospedeiras e o tipo de habitat.

\section{RESULTADOSE DISCUSSÃO}

Foram listadas 12 espécies (Fig. 1), perfazendo 29\% das espécies relacionadas para o Brasil, como segue:

$$
\text { Adelpha Hübner, [1819] }
$$

Adelpha abia (Hewitson, 1850)

Ocorrência. Barracão (Parque Estadual do Espigão Alto), Caxias do Sul, Derrubadas (Parque Estadual do Turvo), Guaíba, Guarani das Missões, Novo Hamburgo, Pareci Novo, Salvador do Sul, Santa Maria, Santa Rosa, Sapucaia do Sul, São Leopoldo e Terra de Areia (BIEZANKo 1949) (CRAM, MAPA, MCNZ e MCTP).

Plantas hospedeiras. Vitex montevidensis (Verbenaceae) (Aiello 1984; Brown Jr. 1992).

Habitat. Florestas mesófilas de encosta, florestas de altitude, campos e moitas floridas (BROwN JR. 1992).

\section{Adelpha calliphana Fruhstorfer, [1913]}

Ocorrência. Rio Grande do Sul (SiLVA et al.1968) (DZUP).

Plantas hospedeiras. Vitex montevidensis (Verbenaceae) (Aiello 1984; BRown JR. 1992).

Habitat. Florestas mesófilas de encosta, florestas de altitude e formações secundárias e antrópicas (BROWN JR. 1992).

Adelpha epizygis Fruhstorfer, [1916]

Ocorrência. Barracão (Parque Estadual do Espigão Alto) e Derrubadas (Parque Estadual do Turvo) (MCNZ e MCTP). Plantas hospedeiras. Vitex sp. (Verbenaceae) (AIELlo 1984). Habitat. Floresta mesófila de encosta entre 800 e 1000 m. Floresta de altitude, geralmente acima de $1100 \mathrm{~m}$, mas se estendendo até $900 \mathrm{~m}$ nos vales. Campos e moitas floridas dentro dos habitats de altitude (BROWN JR. 1992).

Adelpha falcipennis Fruhstorfer, [1916]

Ocorrência. Pelotas e Santa Maria (CRAM, MECB).

Plantas hospedeiras. Vitex sp. (Verbenaceae) (BROwN JR. 1992)

Habitat. Florestas abertas e úmidas, floresta mesófila de encosta, floresta de altitude, campos e moitas floridas (BROwN JR. 1992).

Adelpha goyama Schaus, 1902

Ocorrência. Derrubadas (Parque Estadual do Turvo) (CRAM). Plantas hospedeiras. desconhecidas

Habitat. Floresta estacional decidual.

Adelpha hyas Doyère, [1840]

Ocorrência. Barracão (Parque Estadual do Espigão Alto), Bom Jesus, Cambará do Sul, Caxias do Sul, Derrubadas (Parque Estadual do Turvo), Encantado, Encruzilhada do Sul, Pelotas, Porto Alegre, São Francisco de Paula (Pró-Mata) e Terra de Areia (MABilde 1896; BiezANKo 1949; Bertels \& Baucke 1966; Silva et al. 1968; Teston \& Corseuil 2002) (CRAM, DZUP, MAPA, MCTP, MECB e MRGC).

Plantas hospedeiras. Ilex paraguaiensis (Aquifoliaceae) (BIEZANKo et al. 1974; BROWn JR. 1992) e Casearia silvestris (Flacourtiaceae) (BiEZANKo et al. 1974).

Habitat. Formações antrópicas, florestas, campos baldios, jardins e florestas de eucaliptos ou pinheiros, florestas de altitude e topos de morro cercados de florestas de qualquer tipo (BRown JR. 1992).

Adelpha isis (Drury, 1782)

Ocorrência. Terra de Areia (MCTP).

Plantas hospedeiras. Cecropia sp., Coussapoa sp. (Cecropiaceae) e Pourouma sp. (Moraceae) (MÜLLER 1886).

Habitat. É bastante rara no Rio Grande do Sul, podendo ser vista em topos de morro e na beira de riachos (BROwN JR. 1992).

\section{Adelpha mincia Hall, 1938}

Ocorrência. Barracão (Parque Estadual do Espigão Alto), Derrubadas (Parque Estadual do Turvo), Encruzilhada do Sul, Erechim, Novo Hamburgo, Pelotas, Santa Maria, Santa Rosa, São Luiz Gonzaga e Sarandi (Parque Estadual do Rondinha) (BIEZANKo 1949) (CAMB, CRAM, DZUP, MCTP, MECB e MUCP).

Plantas hospedeiras. Guettarda uruguensis (Rubiaceae).

Habitat. Floresta mesófila de encosta, geralmente entre 800 e $1000 \mathrm{~m}$, campos e moitas floridas em florestas de altitude (BROWN JR. 1992). 


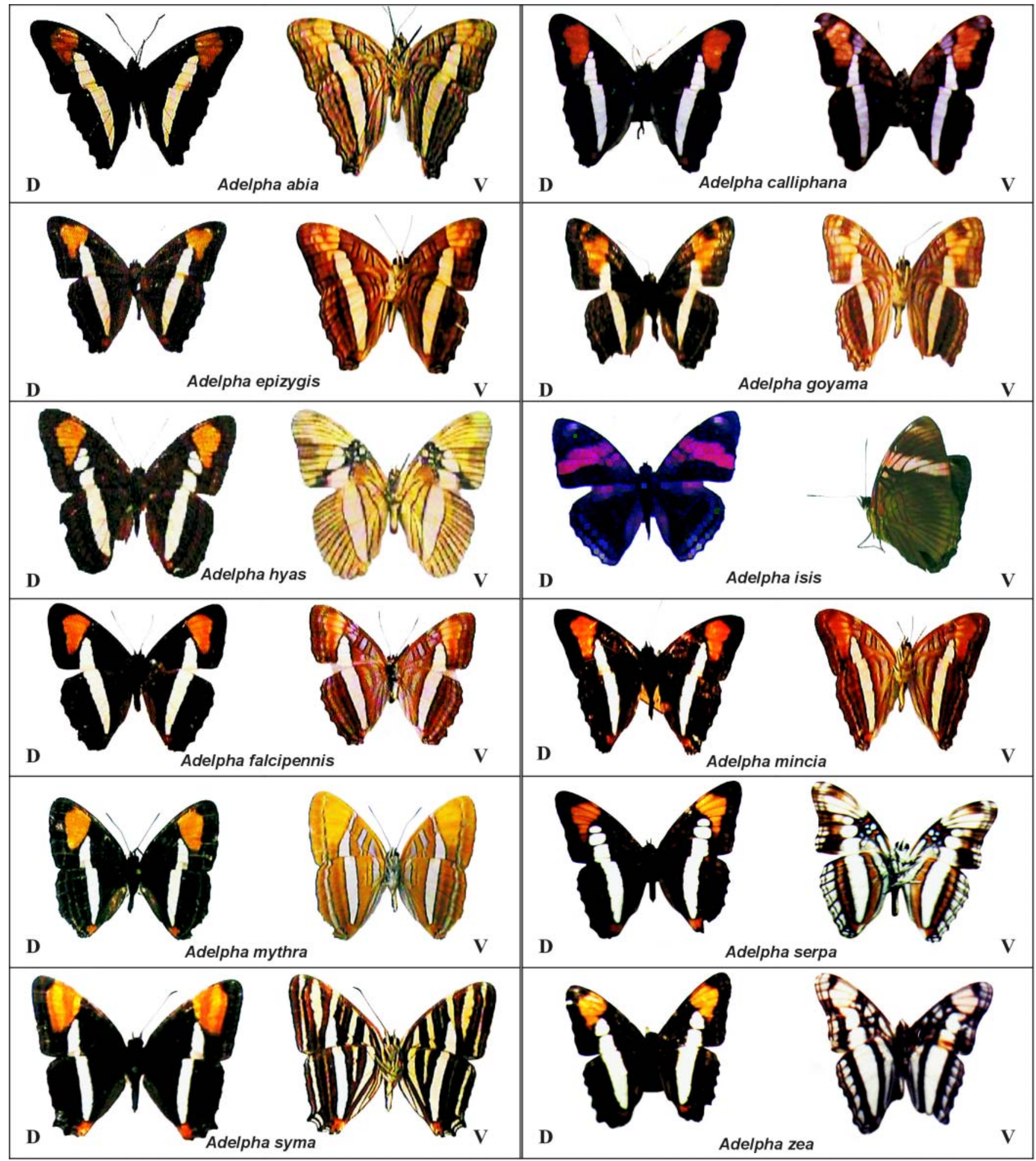

Fig. 1. Espécies de Adelpha ocorrentes no Rio Grande do Sul. D = vista dorsal; V = vista ventral. 
Adelpha mythra (Godart, [1824])

Ocorrência. Barracão (Parque Estadual do Espigão Alto), Campo Bom, Canela, Derrubadas (Parque Estadual do Turvo), Encruzilhada do Sul, Guarani das Missões, Igrejinha, Pelotas, Santa Maria, São Francisco de Paula (Pró-Mata), Sapucaia do Sul e Terra de Areia (Weymer 1894; Mabilde 1896; Biezanko B Freitas 1938; Biezanko 1949; Teston \& CORSEUIL 2002) (CAMB, CRAM, DZUP, MAPA, MCTP, MECB e MUCP).

Plantas hospedeiras. Bathysa sp. (Rubiaceae) (Aiello 1984; BRown JR. 1992), Rubus brasiliensis (Rosaceae).

Habitat. Formações secundárias antrópicas, campos baldios, jardins e florestas de eucalipto ou pinheiros, floresta mesófila de encosta, entre 800 e $1000 \mathrm{~m}$, floresta de altitude, campos e moitas floridas em habitats de altitude (BROwN JR. 1992).

Adelpha serpa (Boisduval, 1836)

Ocorrência. Rio Grande do Sul (SiLva et al. 1968) (DZUP, MAPA, MCNZ e MUCP).

Plantas hospedeiras: Miconia sp. (Melastomataceae) (AIEllo 1984; BROWN JR. 1992).

Habitat. Floresta, até acima de $900 \mathrm{~m}$, formações secundárias e antrópicas, campos baldios, jardins e florestas de eucaliptos e pinheiros (BROWN JR. 1992).

Adelpha syma (Godart, 1823)

Ocorrência. Ana Rech, Barracão (Parque Estadual do Espigão Alto), Campo Bom, Canoas, Carlos Barbosa, Caxias do Sul (Vila Oliva), Derrubadas (Parque Estadual do Turvo), Erechim, Maquiné, Montenegro, Morro Reuter, Nova Petrópolis, Osório, Pareci Novo, Pelotas, Porto Alegre, Santa Maria, São Francisco de Paula (Pró-Mata), Sapucaia do Sul, Terra de Areia e Três Cachoeiras (Weymer 1894; Mabilde 1896; Ronna 1934; BiezANKo \& Freitas 1938; SiLVA et al. 1968; TESTON \& CORSEUIL 2002) (CRAM, DZUP, MAPA, MCTP, MECB, MRGC e MUCP).

Plantas hospedeiras. Rubus sp. (Rosaceae) (Aifllo 1984; BiezANKo et al. 1949; ÔRøžÓUšú et al. 1974), Rubus fructicosus (Rosaceae) (BRown JR. 1992), Cephalanthus glabrathus (Rubiaceae) (Aiello 1984; BiezANKo 1949) e Cephalanthus sarandi (Rubiaceae) (RonNA 1933).

Habitat. É comum em florestas perturbadas. Formações secundárias e antrópicas, floresta mesófila de encosta, entre 800 e $1000 \mathrm{~m}$, campos baldios, jardins e florestas de eucaliptos e pinheiros, florestas de altitude acima de $900 \mathrm{~m}$, campos e moitas floridas em habitats de altitude (BROwN JR. 1992).

Adelpha zea(Hewitson, 1850)

Ocorrência. Barracão (Parque Estadual do Espigão Alto), Encruzilhada do Sul, Igrejinha, Novo Hamburgo, Pareci
Novo, São Leopoldo, São Luiz Gonzaga, Sapucaia do Sul e Terra de Areia (BIEZANKo 1949) (CRAM, DZUP, MAPA e MECB).

Plantas hospedeiras. Ilex paraguaiensis (Aquifoliaceae)

(BiEZANKo et al. 1974).

Habitat. Voa em florestas e áreas com moitas floridas adjacentes.

Chave para as espécies de Adelpha Hübner, [1819] ocorrentes no Rio Grande do Sul

1. Com faixa transversal vermelha na asa anterior .... A. isis Sem faixa 2

2(1). Com veias escuras ou negras intercaladas com linhas também escuras ou negras entre as veias na face ventral da asa .................................................. A. hyas

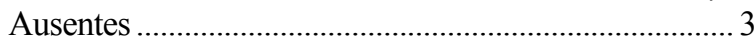

3(2). Faixa branca na asa anterior formada por máculas ....... 4 Faixa branca na asa anterior, contínua ............................ 5

4(3). Duas máculas formam a faixa branca na asa anterior ....... A. zea

Três máculas ............................................................ serpa

5(3). Face ventral da asa com coloração amarelo ouro

Com outra coloração A. mythra

6(5). Mancha amarela e faixa branca na face dorsal da asa anterior superpostas . A. goyama

Não superpostas . 7

7(6). Na face dorsal da asa anterior a mancha amarela e a faixa branca estão unidas . .8

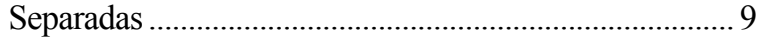

8(7). Mancha amarela na face ventral da asa anterior com recorte anterior dando-lhe um formato em "V" . . A. epizigis

Sem recorte A. abia

9(7). Presença de um conjunto de linhas ou faixas transversais estreitas, brancas e de coloração ferruginosa, com bordas escuras ou negras, na face ventral da asa A. syma

Sem esse conjunto de linhas ou faixas 10

10 (9). Faixa branca na face ventral da asa anterior estende-se até a costa através de uma série de máculas de coloração acinzentada ... 11

Faixa branca não se estende até a costa ............. A. mincia

11(10). Máculas de coloração acinzentada que excedem a largura da faixa branca e separadas por uma linha transversal escura A. falcipennis 
Máculas sem linha transversal escura A. calliphana

O exame dos detalhes da face ventral, quanto às linhas e manchas e, na face dorsal, quanto à junção da mancha amarela com a faixa branca, é bastante útil na diferenciação das espécies.

Adelpha catharina var. Staudinger citada por MABILDE (1896) deixou de ser incluída na listagem, pela insuficiência da caracterização feita por este autor e pelo fato do exemplar existente na coleção Mabilde (MCNZ) ser idêntico à A. zea.

São novos registros para o Rio Grande do Sul: A. epizygis, A. falcipennis, A. goyama e A. isis.

\section{REFERÊNCIAS}

Aiello, A. 1984. Adelpha (Nymphalidae): deception on the wing. Psyche 91(12): 1-45.

Bertels, A. \& O. Baucke 1966. Segunda relação das pragas das plantas cultivadas no Rio Grande do Sul. Pesquisa Agropecuária Brasileira 1: 17-46.

Biezanko, C. M. 1949. Acraeidae, Heliconidae et Nymphalidae de Pelotas e seus arredores. Pelotas, Ed. do Autor, 16p.

Biezanko, C. M. \& O. Baucke. 1948. Nomes populares dos lepidópteros no Rio Grande do Sul. Agros 1: 164-177.

BiezANKo, C. M.; R. E. BeRTHOLDI \& O. BAUCKe. 1949. Relação dos principais insetos prejudiciais observados nos arredores de Pelotas nas plantas cultivadas e selvagens. Agros 2(3): 156-213.

Biezanko, C. M. \& R. G. de Freitas. 1938. Catálogo dos insetos encontrados na cidade de Pelotas e seus arredores Fasc. 1- Lepidópteros. Boletim da Escola de Agronomia Eliseu Maciel 25: 1-32.

Biezanko, C. M. \& D. Link. 1972. Nomes populares dos lepidópteros no Rio Grande do Sul (segundo catálogo). Boletim Técnico Defesa Fitosanitaria da Universidade Federal Santa Maria 4: 1-15.

Biezanko, C. M.; A. Ruffinelli \& D. Link. 1974. Plantas y otras sustancias alimenticias de las orugas de los lepidopteros uruguayos. Revista do Centro de Ciências Rurais 4(2): 107-147.

Biezanko, C. M.; A. Ruffinelli \& D. Link. 1978. Catálogo de lepidópteros do Uruguai. Catalogue of Lepidoptera of the Republic of Uruguai. Revista do Centro de Ciências Rurais 8(supl.): 1-84.

BRown JR., K. S. 1992. Borboletas da Serra do Japi: diversidade, habitats, recursos alimentares e variação temporal, p. 142-187. In: L. P. C. Morellato (org.). Historia Natural da Serra do Japi. Ecologia e preservação de uma área florestal no Sudeste do Brasil. Campinas, UNICAMP/FAPESP, 321p.

De LesSe, H. 1967. Les nombres de chromossomes chez les lépidoptéres rhopalocères neotropicaux. Annales de la Societe Entomologique de France (N.S.) 5(1): 67-136.
De VRIES, P. J. 1985. Hostplant records and natural history notes on Costa Rican butterflies (Papilionidae, Pieridae \& Nymphalidae). The Journal of Research on the Lepidoptera 24(4): 290-333.

Fruhstorfer, H. 1907. Adelpha, p. 510-533. In: A. Seitz (ed.). Die GrossSchmetterlinge der Erde. v. 5: Die Gross-Schmentterlinge des amerikanischen Faunengebietes. Stuttgart, F. Lehman Verlag, $1141 \mathrm{p}$.

Godman, F. D. \& O. Salvin. 1879-1901. Biologia Centrali-Americana. Insecta. Lepidoptera-Rhopalocera. London, Dulau \& Co. vol. I [1879-1886], 487p.; vol. II [1887-1901], 782p.

Hall, A. 1938. On the types of Adelpha (Lep., Nymphalidae) in the Collection of the British Museum. Entomologist 71: 184-187.

Hayward, K. J. 1950. Las especies y formas Argentinas del género Adelpha. Acta Zoologica Lilloana 9: 375-393.

JöRGENSEN, P. 1921. Sobre algunos nuevos enemigos de la yerba-mate, Ilex paraguariensis. Revista de la Sociedad Cientifica del Paraguay 1(1): $27-30$.

Mabilde, A. P. 1896. Guia practica para os principiantes collecionadores de insectos, contendo a descrição fiel de perto de 1000 borboletas com 180 figuras lythographadas em tamanho, formas e desenhos conforme o natural. Estudo sobre a caça, classificação conservação de uma colleção mais ou menos regular. Porto Alegre, Gundlach \& Schuldt, 238p.

Miller, L. D. \& Miller, J. Y. 1970. Notes on two rare Mexican Adelpha and related Central American species (Nymphalidae). Journal of the Lepidopterists Society 24(4): 292-297.

Moss, A. M. 1933. Some generalizations on Adelpha, a neotropical genus of nymphalid butterflies of the group Limenitidi. Novitates Zoologicae 39: 12-30.

MüLLER, F. 1886. Sudamerikanische Nymphalidenraupen: Versuch eines natürlichen Systems der Nymphaliden. Zoologische Jahrbücher 1: 417-678.

RonNA, E. 1933. Catálogo dos insetos até hoje encontrados nas plantas do Rio Grande do Sul. Egatea 18(1/2):47-53; (3):96-100; (4):197202; (5):275-278; (6): 329-334.

Silva, A. G. D’ A.; C. R. Gonçalves; D. M. Galvão; A. J. L. Gonçalves; J. Gomes; N. M. Silva \& L. Simoni. 1968. Quarto catálogo dos insetos que vivem nas plantas do Brasil, seus parasitos e predadores. Tomo 1. Parte 2. Rio de Janeiro, Ministério da Agricultura, 622p.

Teston, J. A \& E. Coersuil. 2002. Borboletas (Lepidoptera, Rhopalocera) Ocorrentes no Centro de Pesquisas e Conservação da Natureza PróMata. 3: Nymphalidae. Divulgações do Museu de Ciências e Tecnologia UBEA / PUCRS 7: 1-208.

Weymer, G. 1894. Exotische Lepidopteren VII. Beitrag zur Lepidopterenfauna von Rio Grande do Sul. Stettiner Entomologische Zeitung 55(10/12): 311-333.

Willmott, K. R. \& J. P. W. Hall. 1999. Taxonomic notes on ecuadorian Adelpha, with the description of two new species and seven new subspecies (Lepidoptera: Nymphalidae: Liminitidinae). Tropical Lepidoptera 10(1): 1-17.

Young, A. M. 1974. Notes on the natural history of a rare Adelpha butterfly (Lepidoptera; Nymphalidae) in Costa Rica high country. Journal of the New York Entomological Society 82: 235-244. 